\title{
Vessel Filtering of Photoacoustic Images
}

\author{
Tanmayi Oruganti*†, Jan G. Laufer ${ }^{\ddagger}$, Bradley E. Treeby*† \\ *Research School of Engineering, Australian National University \\ Canberra, ACT 0200, Australia \\ ${ }^{\ddagger}$ Charitè, Universitätsmedizin Berlin, Julius Wolff Institute \\ Augustenburger Platz 1, 13353 Berlin, Germany
}

\begin{abstract}
This paper investigates the application of the vessel filter proposed by Frangi et al., [MICCAI, LNCS vol. 1496, pp. 130-137, 1998] to photoacoustic images of the vasculature. The filter works by classifying the eigenvalue decomposition of the local Hessian matrix at each image voxel to find tubular structures in the image. A detailed analysis of the algorithm is provided, and the effect of the filters on photoacoustic images is studied using numerical and experimental phantoms. In particular, the impact of the filter on image resolution, feature preservation, and noise is discussed. The vessel filter is then applied to photoacoustic images of the vasculature in mice. The classical Hessian filter is shown to be highly effective at removing noise and highlighting vessels, at the expense of reducing the sharpness of vessel edges.
\end{abstract}

\section{INTRODUCTION}

Photoacoustic tomography is a rapidly developing medical imaging modality that allows visualisation of the vasculature in a non-invasive manner. ${ }^{1} \quad$ Laser pulses with wavelengths belonging to the visible and near infrared ranges are used to irradiate the tissue. At these wavelengths, the light has the ability to penetrate several centimetres, and haemoglobin molecules in the blood are the major chromophores which act as natural contrast agents. This leads to the excitation of these molecules and the optical energy gets transformed into heat energy, causing an increase in temperature and pressure in the tissue. Photoacoustic images are then reconstructed using ultrasound signals received at the tissue surface using an ultrasound transducer array.

Photoacoustic images often contain noise or unwanted features, which can be attributed to several different sources. First, background noise is often introduced by electronics in the acquisition system and the limited sensitivity of the detectors. Second, image artefacts can be produced due to the finite size of the individual detector elements, the limited size of the detection aperture, and assumptions made in the reconstruction algorithm. Third, background signals can be generated due to the absorption of optical energy by chromophores outside the blood vessels, which may not be of interest. These sources of noise make photoacoustic images more difficult to interpret. Consequently, finding robust ways to reduce or eliminate this noise is of interest.

Over the last two decades, various vessel extraction algorithms have been developed for performing vessel segmentation in images acquired using CT and MR angiography. ${ }^{2}$ The algorithms vary depending on the purpose of application, user interaction, computational costs, medical image type, and various other factors. ${ }^{3}$ One of the most widely used algorithms was proposed by Frangi et al. ${ }^{4}$ This filter works by classifying the eigenvalue decomposition of the local Hessian matrix at each image voxel to find tubular structures in the image. Frangi's algorithm has previously been used in several photoacoustic studies, both to reduce noise, ${ }^{5,6}$ to classify vessels for quantitative measurements, ${ }^{7}$ and to extract vessel tortuosity. ${ }^{8}$ Here, we study the algorithm in detail. In particular, the impact of the filter on image resolution, feature preservation, and background noise is discussed.

${ }^{\ddagger}$ T. Oruganti and B. E. Treeby are now with the Department of Medical Physics and Bioengineering, University College London, Gower Street, London WC1E 6BT, UK. Send correspondence to t.oruganti.12@ucl.ac.uk or b.treeby@ucl.ac.uk.

Photons Plus Ultrasound: Imaging and Sensing 2013, edited by Alexander A. Oraevsky, Lihong V. Wang, Proc. of SPIE Vol. 8581, 85811W · C 2013 SPIE · CCC code: 1605-7422/13/\$18 - doi: 10.1117/12.2005988 


\section{OVERVIEW OF FRANGI'S VESSEL FILTERING ALGORITHM}

Blood vessels are approximately tubular or cylindrical structures, and this basic knowledge can be used to derive a filter that classifies vascular features within a 3D image. If the vessel is aligned with the global coordinate system as shown in Fig. 1(a), the curvature or gradient across the vessel (in the $x$ and $z$ directions) will be high, while the curvature along the vessel (in the $y$ direction) will be low. This could be used to classify vessels within an image, however, in general the vessel won't be aligned with the global coordinate system. The basis of Frangi's vessel filtering algorithm is to first find the local coordinate system aligned with the vessel, and then use the curvature in these directions to classify different structures.

The algorithm has several computational steps which are outlined in Fig. 1(b). First, the algorithm performs Gaussian smoothing by convolving the 3D input image with a Gaussian kernel of the appropriate scale $s$ (this is equal to the standard deviation of the Gaussian). A Gaussian function, which is often used as a low-pass filter, is known to have excellent smoothing properties and is used to eliminate high frequency variations in the image, generally associated with noise. The Gaussian kernel at position $\mathbf{x}=\{x, y, z\}$ and scale $s$ is given by

$$
G(\mathbf{x}, s)=\frac{1}{\sqrt{2 \pi s^{D}}} \exp \left(\frac{-\|\mathbf{x}\|^{2}}{2 s^{2}}\right)
$$

and the smoothed image $L(\mathbf{x}, s)$ is computed by

$$
L(\mathbf{x}, s)=L(\mathbf{x}) * G(\mathbf{x}, s),
$$

(the image data is grayscale or scalar rather than RGB). The scale is chosen by the user depending on the size of vasculature present in the image. Since the sizes of the blood vessels typically vary between a range of radii, it is necessary to use multiple scales of the Gaussian kernel to preserve them all. Hence, a multiscale filter is used. Section 3 discusses how the scales are chosen based on the sizes of structures of interest.

After smoothing, an orthogonal coordinate system aligned with local features in the image is found by forming and decomposing the $3 \times 3$ Hessian matrix at every image voxel. The Hessian matrix consists of second order derivatives that contain information about the local curvature. At a particular position $\mathbf{x}$ in the image $L(\mathbf{x}, s)$, the Hessian $\mathcal{H}_{\mathbf{x}, s}$ is given by

$$
\mathcal{H}_{\mathbf{x}, s}=\left[\begin{array}{ccc}
L_{x x}(\mathbf{x}, s) & L_{x y}(\mathbf{x}, s) & L_{x z}(\mathbf{x}, s) \\
L_{y x}(\mathbf{x}, s) & L_{y y}(\mathbf{x}, s) & L_{y z}(\mathbf{x}, s) \\
L_{z x}(\mathbf{x}, s) & L_{z y}(\mathbf{x}, s) & L_{z z}(\mathbf{x}, s)
\end{array}\right],
$$

where $L_{x y}(\mathbf{x}, s)=\frac{\partial}{\partial x}\left(\frac{\partial}{\partial y} L(\mathbf{x}, s)\right)$ and so on. An eigenvalue decomposition is then performed to find the eigenvectors and eigenvalues of the Hessian matrix. As the Hessian is a $3 \times 3$ matrix, there are three eigenvectors at every voxel. The set of eigenvectors represents a new orthonormal coordinate system aligned according to the local curvature. The corresponding eigenvalues quantify the magnitude of the curvature in these directions, and can thus be used to identify vessel-like structures.

To quantify any local structures in the image, the eigenvalues for each voxel are arranged in increasing order such that $\lambda_{1} \leq \lambda_{2} \leq \lambda_{3}$. A vessel can then be identified based on the criteria that the curvature is high in two directions, and low in the third, i.e.,

$$
\left|\lambda_{1}\right| \approx 0, \quad\left|\lambda_{1}\right| \ll\left|\lambda_{2}\right|,\left|\lambda_{3}\right|
$$

In Frangi's vessel filter, several ratios are defined to classify different structures (see Table 1). The first ratio differentiates between vessel-like structures and plate-like structures and is given by

$$
\mathcal{R}_{A}=\frac{\left|\lambda_{2}\right|}{\left|\lambda_{3}\right|}
$$

In the case of a vessel, the two largest eigenvalues (corresponding to the eigenvectors through the vessel crosssection) are large and approximately equal. Comparatively, the eigenvalue $\lambda_{1}$ along the length of the structure 
(a)
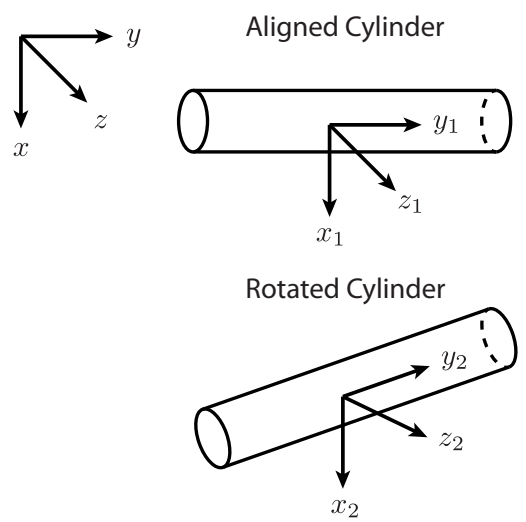

(b)

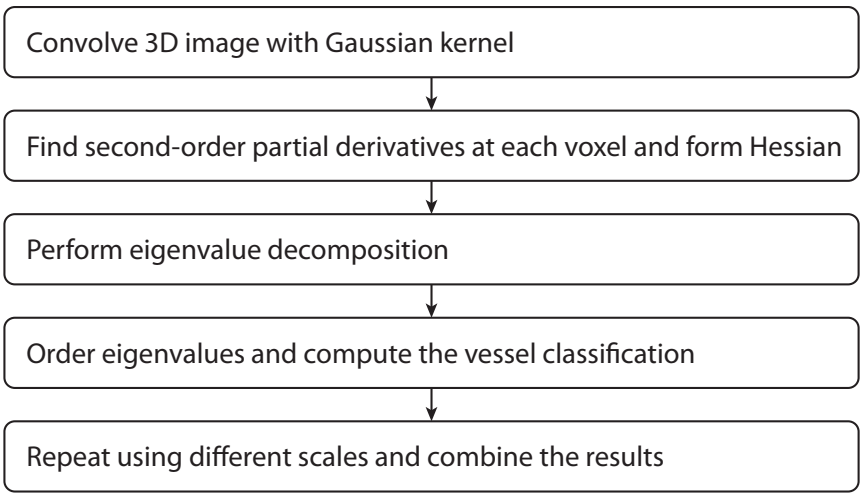

Figure 1. (a) Schematic of an ideal vessel aligned with the global coordinate system (top) and local coordinate system (bottom). The vessel can be classified by examining the curvature in each direction in the local coordinate system. (b) Flow chart summarising the steps in computing Frangi's vessel filtering algorithm.

is small in magnitude. In the case of a plate-like structure, the first two eigenvalues are small and only $\lambda_{3}$ (corresponding to the eigenvector across the thickness of the plate) is of a higher magnitude. These structures can thus be distinguished by comparing the ratio of the largest two eigenvalues. This ratio attains a maximum of 1 for vessel-like structures and a minimum of 0 for plate-like structures.

The second ratio distinguishes blob or ball-like structures and is given by

$$
\mathcal{R}_{B}=\frac{\left|\lambda_{1}\right|}{\sqrt{\left|\lambda_{2}\right|\left|\lambda_{3}\right|}}
$$

Blob-like structures have a high curvature in all three directions with eigenvalues of approximately equal magnitude. These structures can thus be distinguished by comparing the smallest eigenvalue to the largest two. The ratio attains a maximum of 1 for ball or blob-like structures and a minimum of 0 for other types of structures.

The final ratio (called the 'structureness' measure by Frangi) is given by

$$
\mathcal{S}=\|\mathcal{H}\|_{F}=\sqrt{\sum_{j \leq D} \lambda^{2}}=\sqrt{\lambda_{1}^{2}+\lambda_{2}^{2}+\lambda_{3}^{2}}
$$

This measure calculates the Frobenius norm of the matrix, which can separate the structures of interest from noise for a given signal-to-noise ratio. The norm takes into account all three eigenvalues and can distinguish between signal and noise as any structures present in the image will have at least one large eigenvalue.

A combined vesselness measure is then computed by combining the three ratios:

$$
\mathcal{V}(\mathbf{x}, s)= \begin{cases}0, & \lambda_{2}>0 \text { or } \lambda_{3}>0 \\ \left(1-\exp \left(\frac{-\mathcal{R}_{A}^{2}}{2 \alpha^{2}}\right)\right) \exp \left(\frac{-\mathcal{R}_{B}^{2}}{2 \beta^{2}}\right)\left(1-\exp \left(\frac{-\mathcal{S}^{2}}{2 c^{2}}\right)\right), & \text { otherwise }\end{cases}
$$

This equation helps identify tubular or vessel-like structures in an image while eliminating noise and other structures. Here, $\alpha, \beta$ and $c$ are thresholds that control the sensitivity of the filter to vessels, blob-like structures, and noise, respectively. The significance of these parameters and the scale parameter is discussed in Sec. 3.1. The filter also eliminates the structures with positive eigenvalues as these indicate regions of low optical absorption on a background of high optical absorption which is not physical for vasculature within a photoacoustic image. 
Table 1. Summary of geometric shapes associated with different eigenvalue ratios.

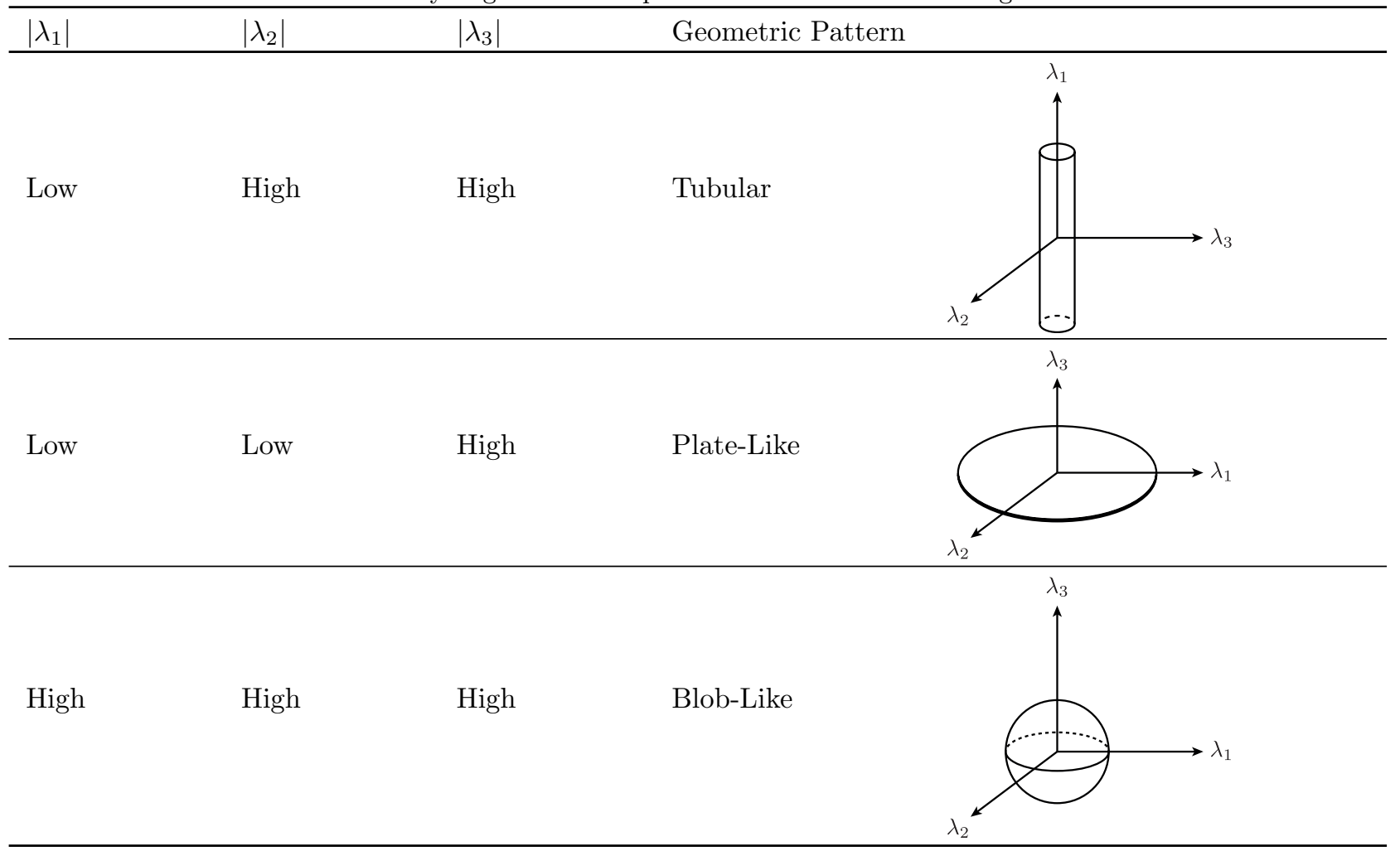

The final step is to apply the vessel filter repeatedly using different Gaussian scales to take into account different vessel sizes within the 3D image. The images at different scales are then combined to give a final multiscale vesselness measure given by

$$
\hat{\mathcal{V}}(\mathbf{x})=\max _{s_{\min } \leq s \leq s_{\max }} \mathcal{V}(\mathbf{x}, s)
$$

Here $\hat{\mathcal{V}}$ is the final multiscale filter output where $\hat{\mathcal{V}} \in[0,1]$. This value corresponds to the likelihood of each image voxel belonging to a vessel.

\section{NUMERICAL EXPERIMENTS}

\subsection{Sensitivity parameters}

The vesselness measure contains three parameters $(\alpha, \beta$ and $c)$ that control the sensitivity of the filter to different geometrical structures present in the image. Following Frangi, the parameter $c$ is set adaptively to half the value of the maximum Hessian norm. The parameters $\alpha$ and $\beta$ can be modified by the user as required. In Fig. 2(a), the first term of the vesselness measure, that which distinguishes between vessels and plate-like structures, has been plotted against the ratio $\mathcal{R}_{A}$ for different $\alpha$ values. It can be observed that increasing the value of alpha reduces the probability of identifying vessels for a given $\mathcal{R}_{A}$. Similarly, in Fig. 2(b), the second term of the vesselness measure has been plotted against the ratio $\mathcal{R}_{B}$ for different $\beta$ values. The $\beta$ parameter controls the sensitivity of the filter to blob or ball-like structures in the image. It can be seen that increasing the value of $\beta$ makes the filter less sensitive to these structures. In other words, if $\beta$ is increased, the penalty for finding a blob-like structure on the vesselness metric is reduced.

\subsection{Selecting the optimal filter scales}

When applying the vessel filter to image data, it is important to realise the significance of choosing the scale of the Gaussian kernel. The question of interest is, what scale should be chosen for a given vessel radius? Firstly, 

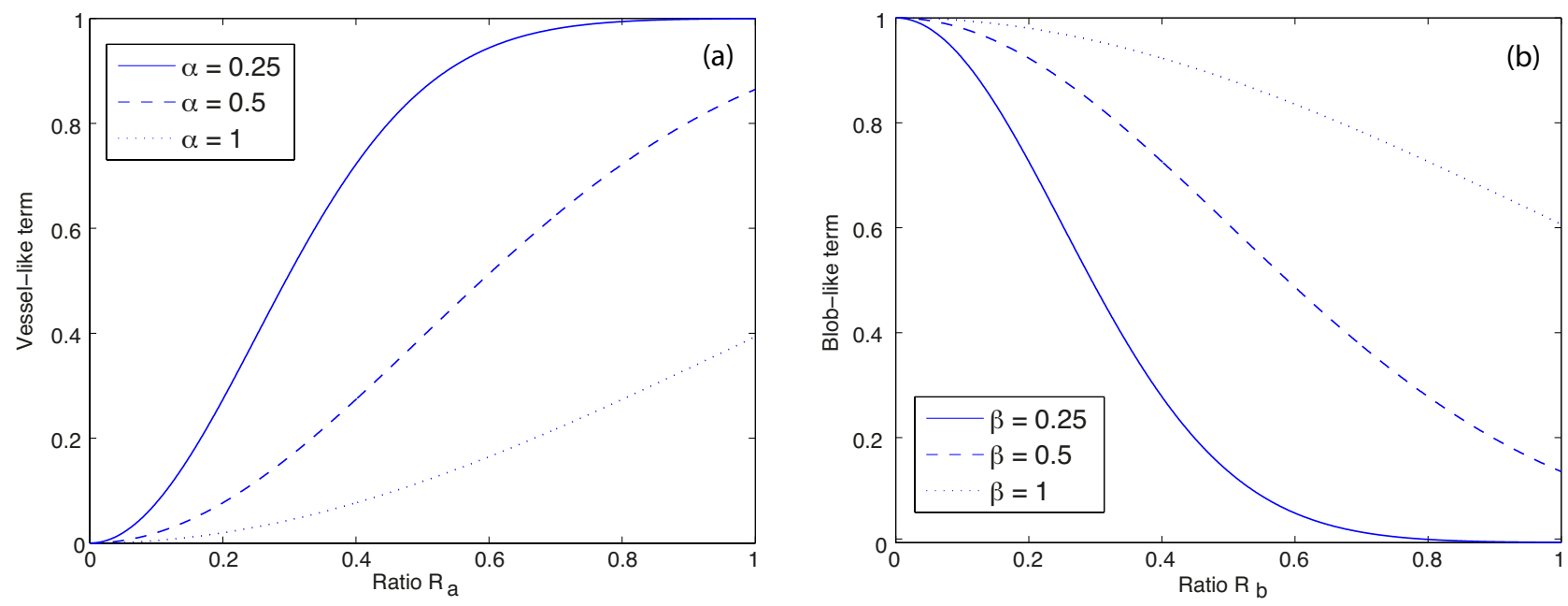

Figure 2. (a) Changes in the tubular or vessel-like term in the vesselness metric with changes in the ratio $\mathcal{R}_{A}$. Changes in the ball or blob-like term in the vesselness metric with changes in the ratio $\mathcal{R}_{B}$.

the limits can be derived. If the scale of the Gaussian kernel is very small compared to the vessel radius, the Gaussian acts like a delta function, and the convolution simply yields the input vessel. When the vesselness metric is calculated, two peaks are generated at each of the edges, predicting more than one vessel in the image and an absence of vessel along the centre as shown in Fig. 3(a). However, the magnitude of the vessel metric is also very low in amplitude. If the scale of the Gaussian kernel is instead very large compared to the vessel radius, the vessel acts as like a delta function, and the convolution yields the Gaussian kernel of the chosen scale in place of the vessel. When the vesselness metric is calculated, the resultant image is a very blurred version of the input image as shown in Fig. 3(b).

Between these limits, the value of the vessel metric will depend on the ratio of the actual vessel width to the filter scale. To determine the optimal value, several numerical experiments were performed using a 3D image containing a perfect cylinder. This image was then filtered at different scales, and the resulting change in the full-width at half-maximum $\left(\right.$ FWHM) and peak values $\left(\mathcal{V}_{\max }\right)$ calculated from a $1 \mathrm{D}$ profile through the vessel cross-section. Figure 4(a) shows the variation in the FWHM and peak values against the scale to radius ratio. It can be observed that both $\mathcal{V}_{\max }$ as well as the FWHM are maximised when the scale is approximately half the size of the radius of the input vessel (cylinder). When the scale is much less than this optimal value, the width of the vessel becomes too big for the filter to detect and instead the filter recognises two vessels at the edges, as shown in the plot. Similarly, when the scale of the filter is bigger than the optimal scale, the blurring of the vessel edges becomes much larger.

In Fig 4(b), a 1D profile through the vessel filtered image is shown for different $\alpha$ values with the filter scale set to half the vessel radius (a profile through the input cylinder is shown in black). It can be noticed that even for the ideal conditions, i.e., a perfect vessel, no background noise or other image features, and the optimal filter scale and alpha values, there is a significant loss of information. In particular, the edges of the vessel are noticeably blurred after applying the vessel filter, and the FWHM is decreased. This information loss makes the filter quantitatively less accurate and can be seen as one of the limitations of the filter.

\subsection{Response to noise}

Noise is one of the major causes of concern in medical images, and vessel enhancement and segmentation filters try to address this problem. Noise, and the image artefacts that could be generated due to noise, can cause difficulty in performing further analysis of the images, as they might be misinterpreted for structures that are not actually present in the original target. To study the filter response to noise, two numerical experiments were performed. In the first case, Gaussian or normally distributed noise was used as the input to the vessel enhancement filter. The vessel filtered outputs were obtained at scale 2 and 5. Maximum intensity projections 

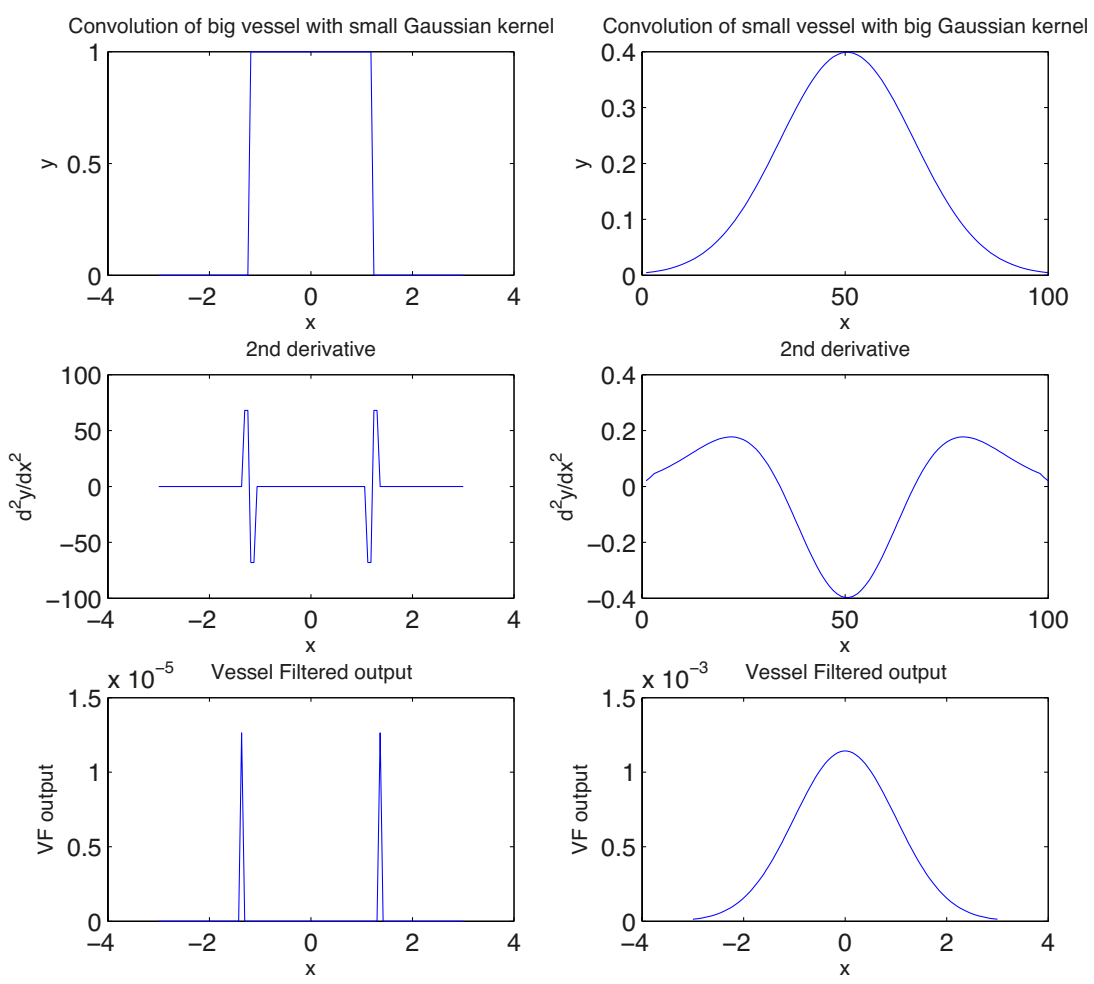

Figure 3. Left (top to bottom): Case when a vessel with a large radius is filtered using a small scale. The three panels show the result of the convolution, 2nd derivative, and the vessel filtered output. Right (top to bottom): Case when a vessel with a very small radius is filtered using a large scale. The three panels again show the result of the convolution, 2nd derivative, and the vessel filtered output.
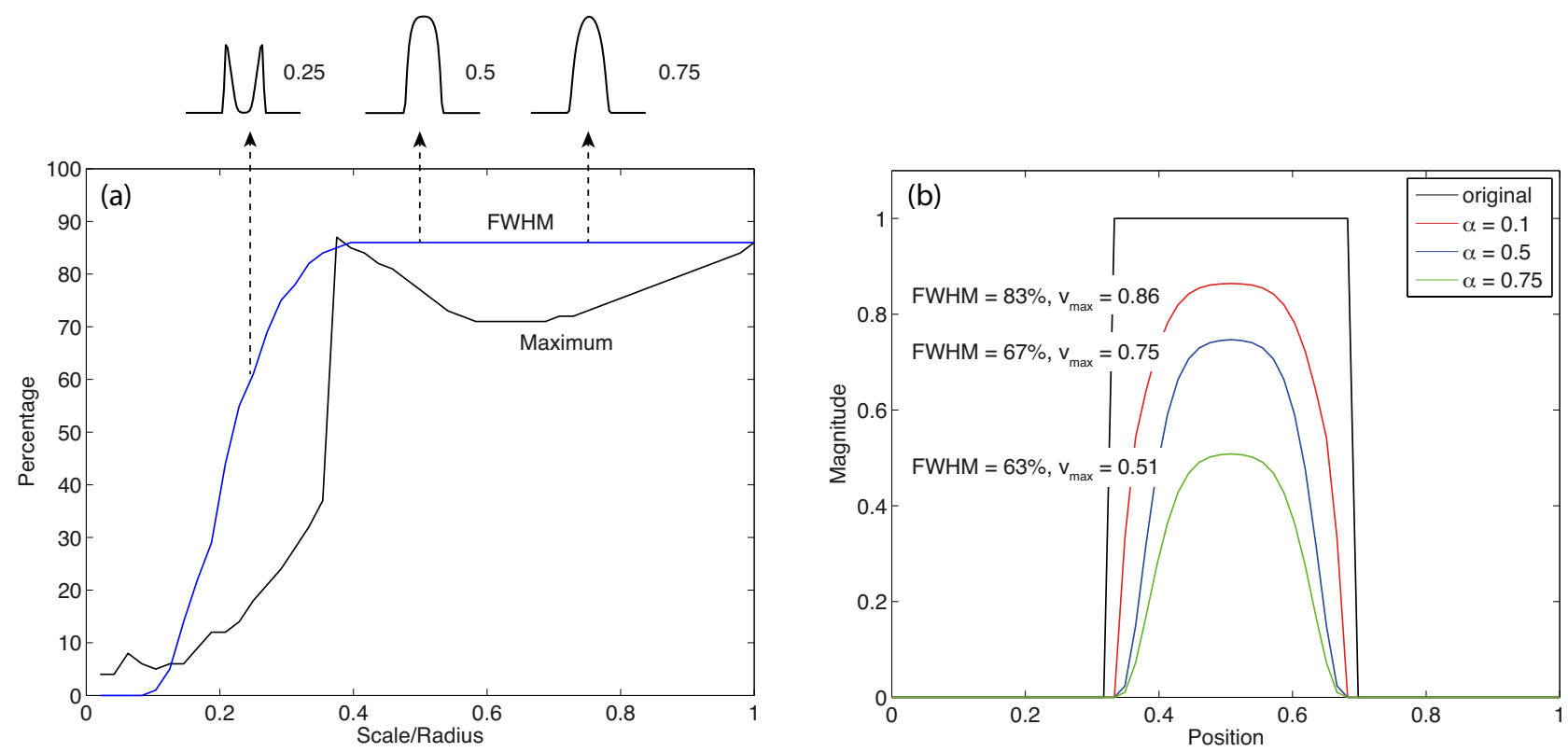

Figure 4. (a) Change in the full-width at half maximum (FWHM) and maximum value of a vessel filtered cylinder with the filter scale. (b) One-dimensional profiles through vessel filtered images of a cylinder for different $\alpha$ values. 
(a)

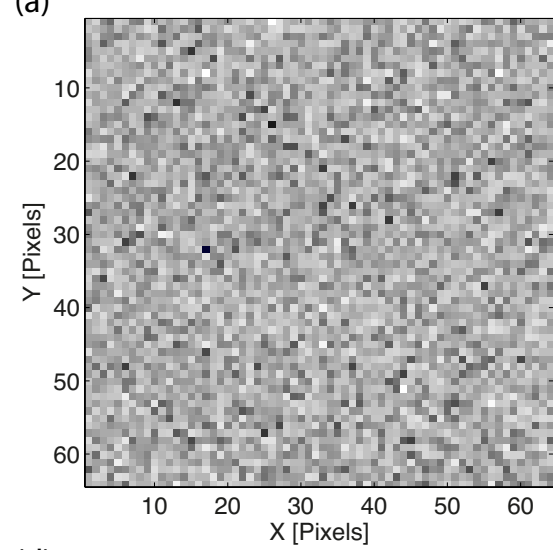

(d)

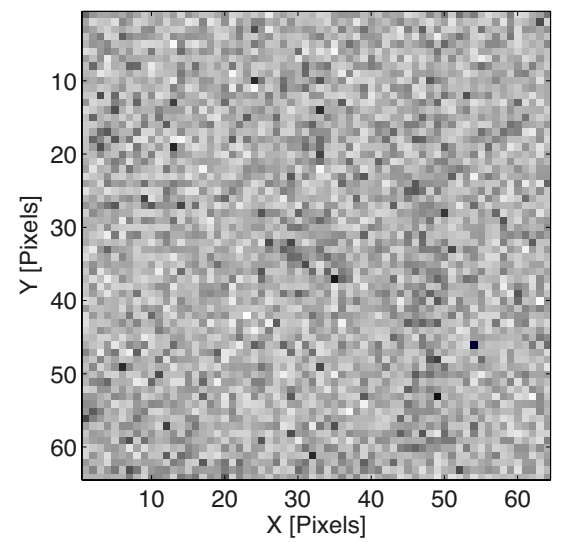

(b)

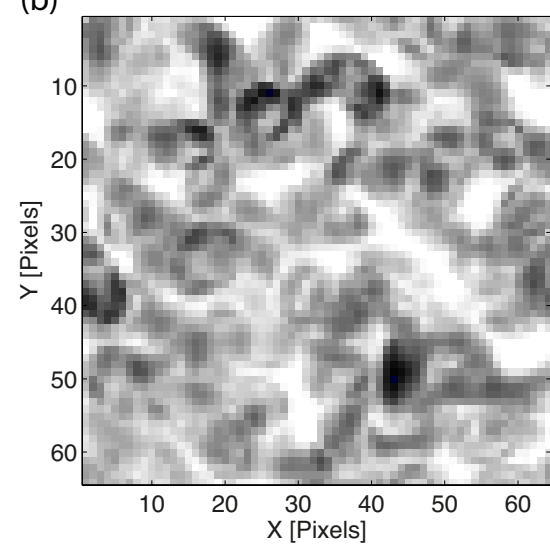

(e)

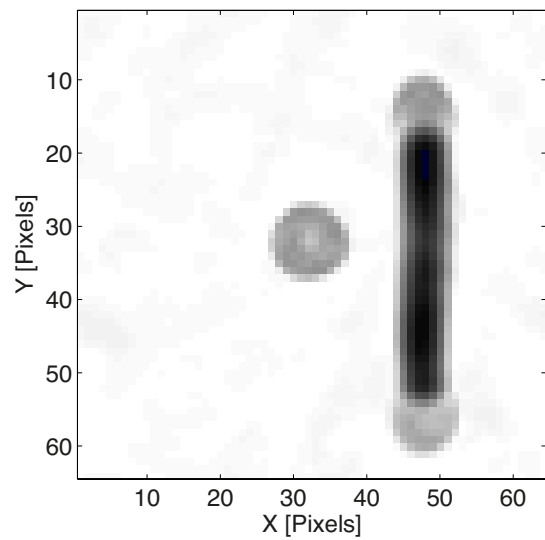

(c)

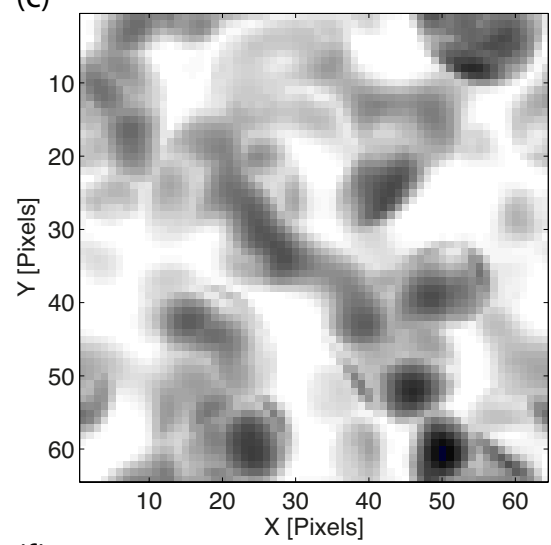

(f)

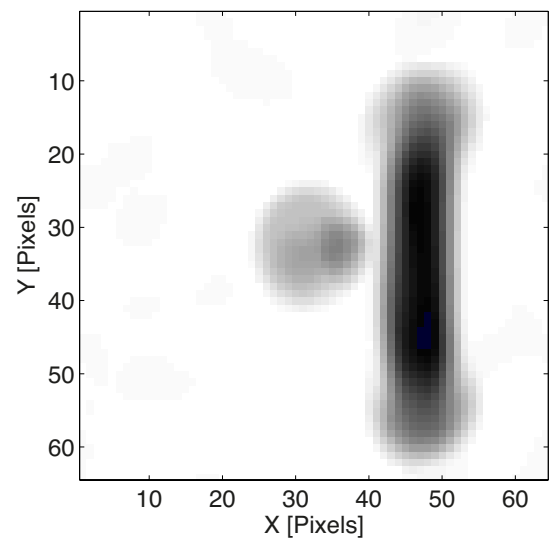

Figure 5. (a) Input image containing Gaussian white noise. (b) Vessel filtered image at scale of 2. (c) Vessel filtered image at scale of 5. (d) Input image containing cylinder (radius $=5$ ), sphere (radius $=5$ ) along with additive white gaussian noise. (e) Vessel filtered image at scale 2. (f) Vessel filtered image at scale of 5.

of the input and output images are shown in Figs. 5(a)-(c). It is clear that the filter generates structures that weren't initially present in the image. However, these structures do not appear vessel-like. The size of the structures is also dependent on the scale of the filter (they are larger in Fig. 5(c) than Fig. 5(b)).

In the second case, the same Gaussian noise was added to an image containing a tubular and spherical structure, each of radius 5. Maximum intensity projections of the input and output images are shown in Figs. 5(d)-(f). In the output images, it can be seen that the noise has been almost completely eliminated, and the spherical structure is largely blurred while the tubular structure is preserved. A significant difference can be observed between the output images shown in Figs. 5(b)-(c) compared with Figs. 5(e)-(f), despite the same Gaussian noise being present in both images. Hence, at least for Gaussian noise, it can be seen that the filter only generates artefacts in the absence of any other structures. Conversely, in the presence of actual structures, a clear distinction can be made between signal and noise.

\section{APPLICATION TO PHOTOACOUSTIC IMAGES}

The previous sections give some insight into the mathematical background and characteristics of the vessel filter. To examine the performance of the filter in the context of photoacoustic tomography, the filter was also applied to reconstructed photoacoustic images acquired using a planar tomography system based on a high-sensitivity Fabry-Perot sensor. ${ }^{9}$ The first image was a 3D photoacoustic image of blood filled tubes in a $1.5 \%$ solution of intralipid first presented by Zhang et al. ${ }^{9}$ Depth-direction maximum intensity projections through the original and vessel filtered images are shown in Fig. 6. In the original image, there is a significant 

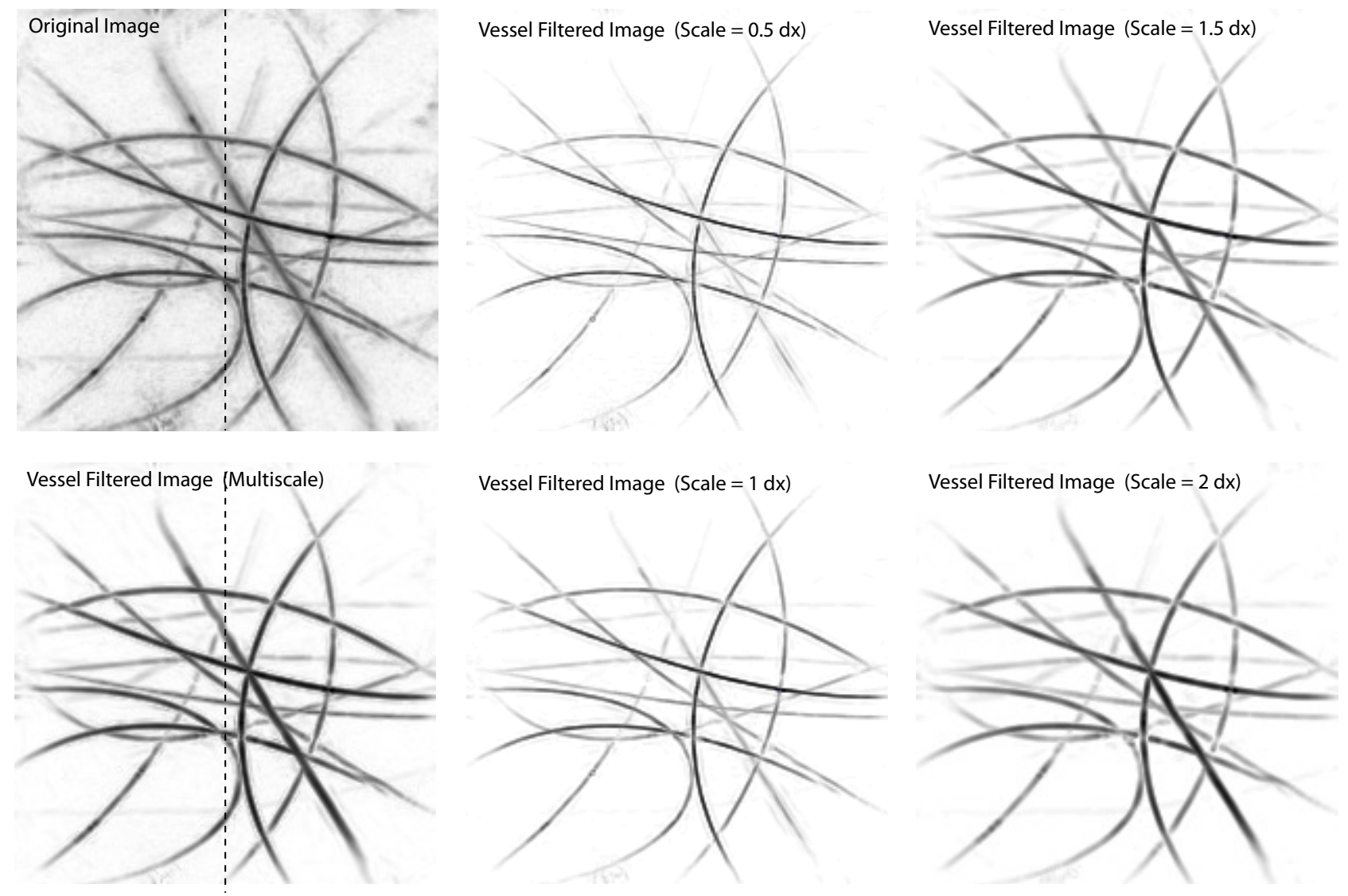

Figure 6. Depth-direction maximum intensity projections through a $3 \mathrm{D}$ photoacoustic image of several blood filled tubes immersed in intralipid. The original image is shown in the top left panel, with the vessel filtered image at various scales shown in the remaining panels.

amount of noise present. It can also be observed that there are various sizes or widths of vessels present, all of which need to be enhanced by the filter. The filter was applied to the image at various scales, starting from a scale of 0.5 to 2 .

At the lower filter scale, vessels of smaller radii are better preserved. As the scale is increased, the thicker vessels are highlighted. Comparing the final multiscale image with the input image, it can be seen that the filter has successfully eliminated noise in the image, and significantly improved the image contrast. No additional artefacts have been introduced, and all the tubes/vessels present in the original image have been preserved and enhanced. Figure 7 shows normalised 1D profiles through the original and the multiscale vessel filtered images (the location of the profile is shown with the dashed lines in Fig. 6). This illustrates a reduction in the noise floor (or background noise level) which has increased the signal-to-noise ratio and image contrast in the vessel filtered output.

A second example of the vessel filter applied to a 3D photoacoustic image is shown in Fig. 8. In this case, the image is of the vasculature in the flank of an adult mouse. The imaging protocol used to acquire this dataset is described in detail in Laufer et al. ${ }^{10}$ The vessel filtered has preserved all the vessels present in the original image, and the filtered image shows a great improvement in the image contrast. Again, the background noise has been removed and a clear distinction between vessel and non-vessel regions can be made based on the vessel filtered image. In several regions of the image, it is also much easier to discern small vessels from the background. 


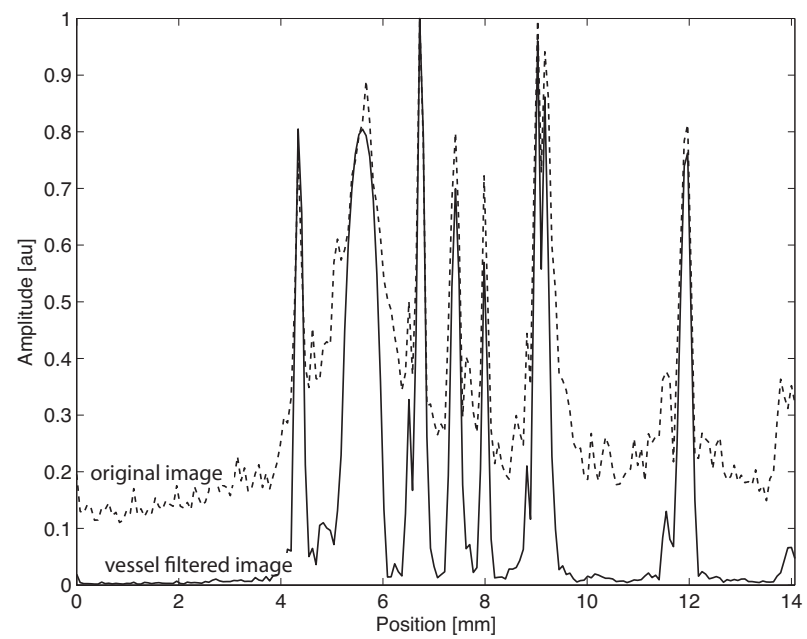

Figure 7. One-dimensional profiles through the tube images shown in Fig. 6 . The vessel filter significantly reduces the noise in the image and improves the image contrast.
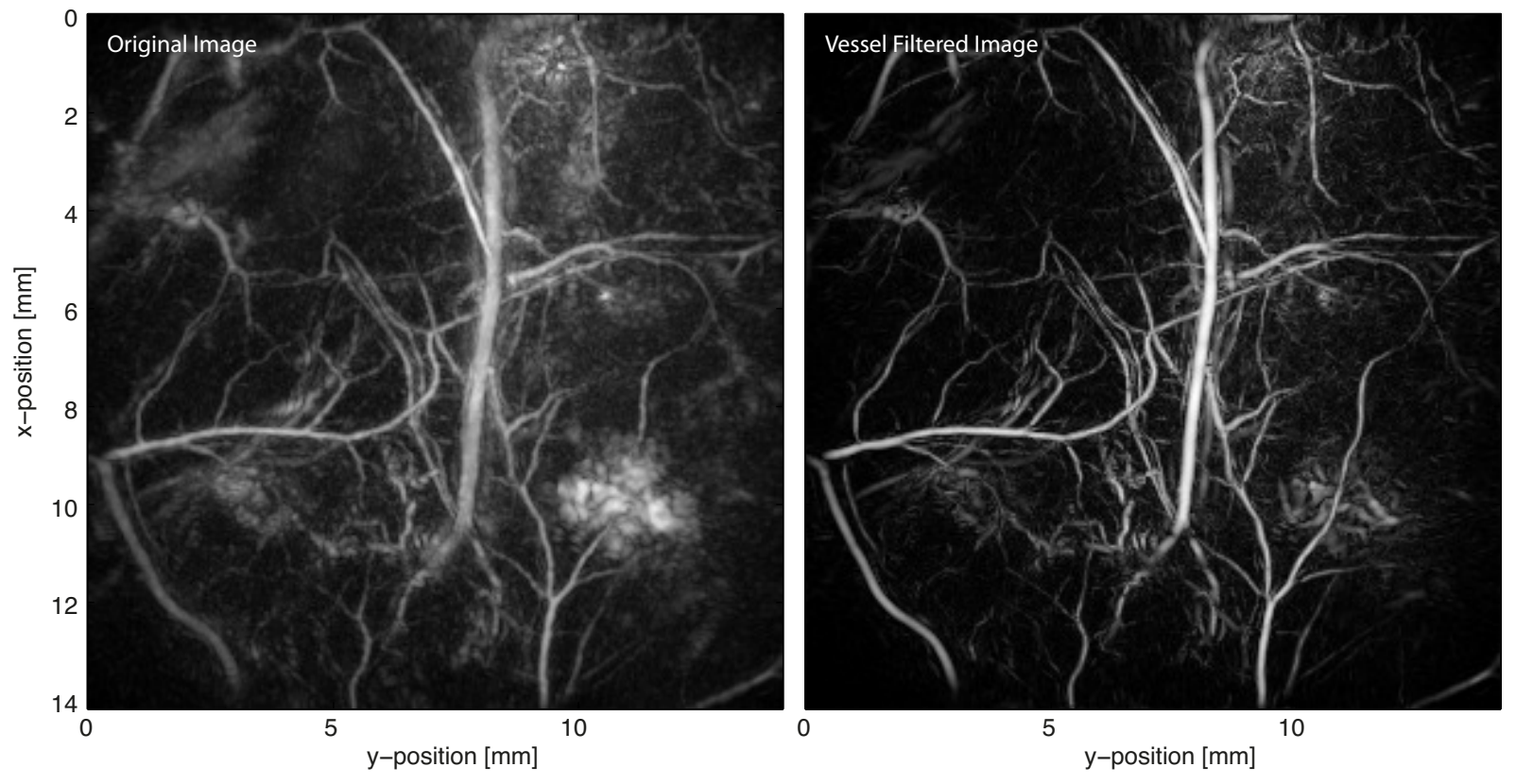

Figure 8. Depth-direction maximum intensity projections through a $3 \mathrm{D}$ photoacoustic image of the vasculature in a mouse flank. The left panel shows the original image, and the right panel the image after multiscale vessel filtering.

\section{DISCUSSION AND CONCLUSION}

Frangi's multiscale vessel enhancement filter, which was originally developed for MR and CT angiography images, was studied and applied to photoacoustic images of the vasculature. Several numerical experiments were performed in order to understand the significance of the different parameters which affect the vesselness metric. It was found that a scale of approximately half the vessel radius maximises the filter response. Similarly, it was found that the vessel filter does not produce artefacts (in particular vessel-like artefacts) in the presence of Gaussian noise if other structures are present in the image. The filter effectively removes background noise and improves the image contrast. However, this come at the expense of smoothing the vessel edges and reducing the FWHM. 
Although the filter is convincing for qualitatively improving photoacoustic images and performing vessel segmentation, directly using the vessel filtered image (for example, by multiplying the original image by the vessel metric, or by using the metric as a binary classifier) for quantitative analysis may not be accurate. This is because the magnitude of the photoacoustic image is related to the local concentration of haemoglobin or other chromophores, thus perturbing the image magnitude will alter the value of any quantitative estimates. The reason for this perturbation is because the Gaussian kernel used to filter the image uniformly smoothes the image, and while doing so, the edges of the vessel become blurred. It is possible that other vessel filtering algorithms that perform anisotropic smoothing could alleviate this problem. ${ }^{11}$

Since the filter recognises vessels based on their geometry, it is possible that the filter could also incorrectly categorise certain image artefacts as vessels (for example, limited-view reconstruction artefacts). This has still not been verified and will be examined as part of future work. It may also be possible to include other metrics into the vesselness measure, for example, information about blood flow rate or oxygen saturation which also indicate the presence of a vessel to improve the robustness of the filter in the context of photoacoustics.

\section{ACKNOWLEDGMENTS}

This work was supported in part by the Australian Research Council/Microsoft Linkage Project LP100100588. The authors would like to thank Yipeng Hu, Dean Barratt, and Eric Rijkhorst from the Centre for Medical Image Computing at University College London for providing initial code and motivation for this work, and Ben Cox and Paul Beard for helpful discussions. The implementation of Frangi's vessel filter used for the study will be released as part of the open-source k-Wave Toolbox (http://www.k-wave.org). ${ }^{12}$

\section{REFERENCES}

1. P. Beard, "Biomedical photoacoustic imaging," Interface Focus 1(4), pp. 602-631, 2011.

2. D. Lesage, E. D. Angelini, I. Bloch, and G. Funka-Lea, "A review of 3D vessel lumen segmentation techniques: models, features and extraction schemes.," Med. Image Anal. 13(6), pp. 819-45, 2009.

3. C. Kirbas and F. Quek, "A review of vessel extraction techniques and algorithms," ACM Computing Surveys 36(2), pp. 81-121, 2004.

4. A. F. Frangi, W. J. Niessen, K. L. Vincken, and M. A. Viergever, "Multiscale vessel enhancement filtering," in Medical Image Computing and Computer-Assisted Interventation (MICCAI), 1496, pp. 130-137, Springer, 1998.

5. W. Shi, P. Hajireza, P. Shao, A. Forbrich, and R. J. Zemp, "In vivo near-realtime volumetric opticalresolution photoacoustic microscopy using a high-repetition-rate nanosecond fiber-laser.," Optics Express 19(18), pp. 17143-50, 2011.

6. Z. Deng, X. Yang, H. Gong, and Q. Luo, "Adaptive synthetic-aperture focusing technique for microvasculature imaging using photoacoustic microscopy," Optics Express 20(7), pp. 11-17, 2012.

7. H. F. Zhang, K. Maslov, M.-L. Li, G. Stoica, and L. V. Wang, "In vivo volumetric imaging of subcutaneous microvasculature by photoacoustic microscopy.," Optics Express 14(20), pp. 9317-23, 2006.

8. S. Hu, C. Favazza, and L. V. Wang, "Multiparameter photoacoustic tomography," in Emerging Imaging Technologies in Medicine, M. A. Anastasio and P. J. La Riviere, eds., pp. 103-119, CRC Press, Boca Raton, 2013.

9. E. Zhang, J. Laufer, and P. Beard, "Backward-mode multiwavelength photoacoustic scanner using a planar Fabry-Perot polymer film ultrasound sensor for high-resolution three-dimensional imaging of biological tissues," Appl. Optics 47(4), pp. 561-577, 2008.

10. J. Laufer, P. Johnson, E. Zhang, B. Treeby, B. Cox, B. Pedley, and P. Beard, "In vivo preclinical photoacoustic imaging of tumor vasculature development and therapy," J. Biomed. Opt. 17(5), p. 056016, 2012.

11. R. Manniesing, M. A. Viergever, and W. J. Niessen, "Vessel enhancing diffusion: A scale space representation of vessel structures.," Med. Image Anal. 10(6), pp. 815-25, 2006.

12. B. E. Treeby and B. T. Cox, "k-Wave: MATLAB toolbox for the simulation and reconstruction of photoacoustic wave fields," J. Biomed. Opt. 15(2), p. 021314, 2010. 